\title{
Erratum to: The intracellular carboxyl tail of the PAR-2 receptor controls intracellular signaling and cell death
}

\author{
Zhihui Zhu $^{1} \cdot$ Rolf Stricker $^{1} \cdot$ Rong yu Li ${ }^{1}$ - Gregor Zündorf ${ }^{1} \cdot$ Georg Reiser $^{1}$
}

Published online: 26 May 2016

(C) Springer-Verlag Berlin Heidelberg 2016

\section{Erratum to: Cell and Tissue Research 359 (3): 817-827, 2015 DOI 10.1007/s00441-014-2056-9}

The authors regret that in our published paper entitled "The intracellular carboxyl tail of the PAR-2 receptor controls intracellular signaling and cell death." Cell and Tissue Research 359 (3): 817-827, 2015; the amino acid sequence given in Figure 6 and hence described in the text had 2 errors. We erroneously omitted one serine $(\mathrm{S})$ in the triple $\mathrm{S}^{383}$ $\mathrm{S}^{384} \mathrm{~S}^{385}$; and, moreover, between $\mathrm{S}^{376}$ and $\mathrm{N}^{377}$ there was an erroneous additional phenylalanine (F). The full length coding sequence of rat PAR-2 (GenBank accession \# U61373.1) contains 397 amino acids.

The error relates to the sequence alignments given in Figure 6, but the nomenclature for the truncation mutants is correct (see below). The error does not affect the generated mutant, or interpretation of the results or the scientific conclusions of the article in any way, because the truncation mutants were correctly expressed and gave the biological responses shown.

The authors would like to apologize for any inconvenience made by this mistake.

The online version of the original article can be found at http://dx/doi.org/ 10.1007/s00441-014-2056-9.

Electronic supplementary material The online version of this article (doi:10.1007/s00441-016-2420-z) contains supplementary material, which is available to authorized users.

Georg Reiser

georg.reiser@med.ovgu.de

Institut für Neurobiochemie, Otto-von-Guericke-Universität Magdeburg, Medizinische Fakultät, Leipziger Straße 44, 39120 Magdeburg, Germany
Therefore, in our article we make the following corrigendum in the text:

1) Replacement for Figure 6 showing the correct sequence and numberings is displayed below. Consequently, the text has the following changes (for the changes, see underlined text).

2) Page 820, Results, ("Construction of PAR-2 truncation mutants"), last 3 sentences:

"From the PAR-2 carboxyl tail sequence $\mathrm{V}^{347}$ SKDFRDQARNALLCRSVRTVKRMQISLTSNKFSRKSSS $\underline{\text { SYSSSSTSVKTSY }}{ }^{397}$ the truncations were generated. The resulting mutants obtained were designated 348-Del, 361-Del, 368-Del, 379-Del, and 386-Del. The number at the respective mutant indicates the first amino acid residue of the deleted part of the receptor. For example, in 348-Del the Cterminal amino acid residue of the truncation mutant is $\mathrm{V}^{347}$, in 361-Del it is $\mathrm{L}^{360}$.

3) Pages 822 and 824, "Ser 349" should read "Ser 348", in all cases (once and 5-times, respectively).

4) Page 826, paragraph 2: "amino acids 387 - 397" should read "amino acids 386 - 397".

5) In addition, the presentations of 4 primers had errors. Thus, corrections on page 818 , last paragraph, for mutant 361-Del the reverse primer is 5'-CTGC AGAA TTCG AAGCT TGAG GAGC GCGT TTCT GGC-3'; on page 819, left column, paragraph 1 , for mutant 379 -Del the forward primer is 5'- TCGC TCAC CTCC AACA AGAA GCTTC GAAT TCTG CAG-3', for mutant 379-Del the reverse primer is 5'-CTGC AGAA TTCG AAGC TTCT TGTT GGAG GTGA GCGA-3'; and for mutant 386-Del the forward primer is 5'-TCCA GGAA ATCC AGCT CTAA GCTT CGAA TTCT GCAG-3'. 
The complete relevant C-terminal nucleotide sequence for rat PAR-2 and the locations for all primers used are given as supplemental information.
We acknowledge the help of Prof. Dr. Ungefroren (University of Lübeck, Germany) by carefully reading our paper and indicating the error therein, as has been corrected here.

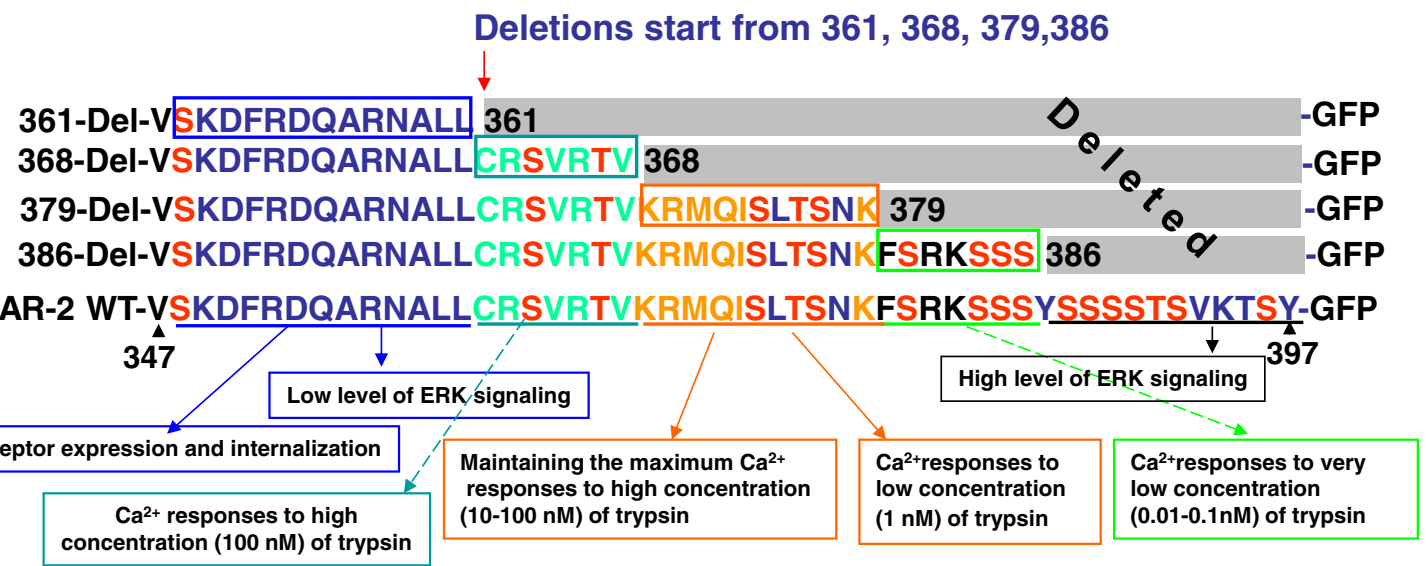

Fig. 6 Functions of different fragments in PAR-2 carboxyl tail truncation mutants. Amino acids in red show the clusters of serines and threonines in PAR-2 carboxyl tail. The amino acid residues 348-360 are supposed to be important for localizing the receptor on the plasma membrane, internalization and signaling to ERK pathway. The amino acid residues 361-367 are suggested to be responsible for $\mathrm{Ca}^{2+}$ signaling to the high concentration of trypsin $(100 \mathrm{nM})$. The amino acid residues $36 \underline{8}-37 \underline{3}$ are important for the $\mathrm{Ca}^{2+}$ responses with the low concentration of trypsin (1 $\mathrm{nM}$ ) and maintaining the maximum $\mathrm{Ca}^{2+}$ responses to the high trypsin concentration. The amino acid residues 379-385 account for the $\mathrm{Ca}^{2+}$ responses with the very low concentrations of trypsin (0.01-01 nM). Amino acids 386-397 (348-360) control the high (low) level of ERK phosphorylation upon trypsin challenge 